\title{
On the Distributed DoS Attacks Targeting at the Bottlenecked Links
}

\author{
More Threatening DoS Attacks than Server Targeting DoS Attacks
}

\author{
Shigeo Akashi \\ Department of Information Sciences, Faculty of Science \\ and Technology, Tokyo University of Science, Noda City, \\ Chiba Prefecture, Japan \\ akashi@is.noda.tus.ac.jp
}

\begin{abstract}
The modern dynamic routing protocols are constructed so as to prevent any routing loops from being brought about spontaneously, because unnecessary circulation of packets on a certain network, whose destinations do not exist unnecessarily consumes the bandwidth which is assigned for the network. In the former half of this paper, we point out the fact that, if we apply dynamic routing together with static one simultaneously, another type of network traffic congestion, which resembles what is brought about by the routing loops, may happen spontaneously. In the latter half of this paper, we discuss the problem asking if this network traffic congestion can be brought about not only spontaneously but also intentionally.
\end{abstract}

\section{CCS CONCEPTS}

- Computing methodologies; • Machine learning; • Machine learning approaches;

\section{KEYWORDS}

Distributed DoS attack, Routing loop, Packet oscillation, Dynamic routing, Static routing, Network traffic congestion, DNS reflection attack.

\section{ACM Reference Format:}

Shigeo Akashi and Yao Tong. 2021. On the Distributed DoS Attacks Targeting at the Bottlenecked Links: More Threatening DoS Attacks than Server Targeting DoS Attacks. In 2021 2nd International Conference on Computing, Networks and Internet of Things (CNIOT 2021) (CNIOT2021), May 20-22, 2021, Beijing, China. ACM, New York, NY, USA, 7 pages. https://doi.org/10.1145/3468691.3468736

\section{INTRODUCTION}

The modern dynamic routing protocols such as OSPF, EIGRP and RIP have been succeeding in protecting our network infrastructures from traffic congestions which are brought about by routing loops. Actually, it sometimes happens that the network traffic has been

Permission to make digital or hard copies of all or part of this work for personal or classroom use is granted without fee provided that copies are not made or distributed for profit or commercial advantage and that copies bear this notice and the full citation on the first page. Copyrights for components of this work owned by others than ACM must be honored. Abstracting with credit is permitted. To copy otherwise, or republish, to post on servers or to redistribute to lists, requires prior specific permission and/or a fee. Request permissions from permissions@acm.org.

CNIOT2021, May 20-22, 2021, Beijing, China

(C) 2021 Association for Computing Machinery.

ACM ISBN 978-1-4503-8969-3/21/05 ..\$15.00

https://doi.org/10.1145/3468691.3468736

\section{Yao Tong}

Department of Information Science, Faculty of Science and

Technology, Tokyo University of Science, Noda City, Chiba

Prefecture, Japan

6320519@ed.tus.ac.jp

suspended by a large number of packets streaming upward and downward simultaneously on the bottlenecked links connecting between the Internet and the gateways of autonomous systems. Though many network administrators are apt to regard these network traffic congestions as spontaneous ones which can be brought about by the fact that the allocated bandwidths are too small to realize smooth packet transfer, we cannot confirm that all the network traffic congestions are brought about spontaneously. In other words, it may be possible that some of network traffic congestions are brought about intentionally.

In the first part of this paper, we introduce classification of cyber attacks from the various kinds of network theoretic viewpoints. It is well known that there are two ways of developing new malicious network skills which can be applied to committing new cybercrimes, one of which is finding security halls existing in our modern network and the other of which is combining network skills with other ones to produce new hybrid cyber attacks. Therefore, it seems to be important that we clarify mutual relations among several cyber attacks and specify the network skills which have been abused for committing these attacks. The authors confide that such classification leads us to the systematization of the cyber attacks and the specification of network skills can contribute to preventing network users from potential cyber attacks before they happen, In the second part of this paper, we introduce the fact that there exist the phenomena that some packets can commute spontaneously on a bottlenecked link between one end and the other one, until their TTLs expire, These phenomena can be called packet oscillations, and in the third part of this paper, we discuss the problem asking if the modern dynamic routing protocols can prevent these packet oscillations. Since it is well known that almost all cyber attacks can be classified into two cases, namely the case consisting of the attacks realized from remote network segments and the case consisting of the attacks realized from local network segments and that the former case is more difficult than the latter case to be realized., in the final part of this paper, we discuss the problem asking if the packet oscillations can be brought about intentionally from remote network segments.

As for the survey of cyber security, we can refer to [1] and [2]. As for the mathematical aspects, we can refer to [3].

\section{CLASSIFICATION OF CYBER ATTACKS}

There exist various kinds of modern cyber attacks bringing about many network failures over the Internet. Actually, the countermeasures preventing authenticated network users from being attacked 
by these attacks seem to be case-by-case or adhoc, Therefore, it is much better for the authenticated network users to be provided with more generic and integrated countermeasures having universal effects on the Internet. This is the reason why we had better classify modern cyber attacks from the point of view of the network theory and investigate the mutual relations between a certain cyber attack and another one. Here we can see several network theoretic criteria as stated below:

Case 1. Whether the cyber attacks originate in the local areas or the remote areas.

It is said that about seventy-five percent of cyber attacks originate in the network segments where the cyber attackers and the sufferers co-exist, because it is quite easy for the cyber attackers not to need to take malicious actions through firewalls and other intrusion detection systems. For example, DHCP spoofing can be classified into two cases, namely DHCP spoofing from inside and DHCP spoofing from outside, the former of which means that the authenticated DHCP client and the malicious DHCP server share the network segments with each other and the latter of which means that the gateway router exists between the malicious DHCP server and the authenticated DHCP client. As for the DHCP spoofing, we can refer to [4]

Case 2. Which layer of the OSI Reference Model is used.

It is not well known that there are several ways with which the cyber attackers take malicious actions though the purpose of the cyber attacks are common among the attackers. For example, phishing fraud can be classified into two case, namely the case which is based on the way of sending malicious e-mail address for replying and the case which is based on the way of DNS spoofing. From the point of view of the OSI Reference Model, the former case is executed with the network skills belonging to Layer 7 and the latter case is executed with the network skills belonging to Layer 3 and Layer 4. This example tells us that some of the cyber attacks can be brought about by applying various kinds of network skills which are different from each other.

Case 3. Whether a cyber attack can evolve more malicously or not.

It is known that Synflood attacks can be more threatening than simple DoS attacks such as Windows F5 attacks, because the cyber attackers continue to send a large number of TCP packets unnecessarily while aborting three-way handshake halfway and forcing port-opening timespan assigned for webservers intentionally long. Moreover, Distributed DoS attacks can be more threatening than simple DoS attacks, because the cyber attackers can take remote control of a large number of PCs over the world and force them to send a large number of TCP packets or UDP packets unnecessarily at the same time.

Case 4. Possibility of the joint combination of a certain cyber attack with another one for the purpose of making new synergetic effects.

As stated in Case 3, there are various ways, which can make DoS attacks more threatening, and each of these ways is different from the other ways. For example, Synflood attacks can be executed by applying TCP three-way handshake maliciously, while Distributed DoS attacks can be executed by the network skills which is analogous to Windows Remote Desktop Connection. Moreover, since the network skills used for Synflood attacks is compatible with the network skills used for Distributed DoS attacks, it implies that the cyber attackers can construct the hybrid type of DoS attacks, which should be called Distributed Synflood attacks. This consideration tells us that it is important for the network administrators not only to specify the network skills having abused for commiting cyber attacks but also to investigate the compatibility of a certain abused network skill with another abused one, The same consideration as stated above can be applied to some other cyber attacks than DoS attacks.

\section{NETWORK TRAFFIC CONGESTONS}

In the previous section, it is assumed that the cyber attackers executing DoS attacks target servers or PCs. In this section, we discuss the problem asking if there is another cyber attacks which can bring about the network traffic congestions in the way of targeting any other network instruments than the servers, because DoS attacks can also bring about the network traffic congestions. Exactly speaking, intentional network traffic congestions can be classified according to a difference in the destinations which the cyber attackers target. Here we assume that the topological relations among the cyber attackers, the servers suffering from attacks and the Internet are illustrated as the following:

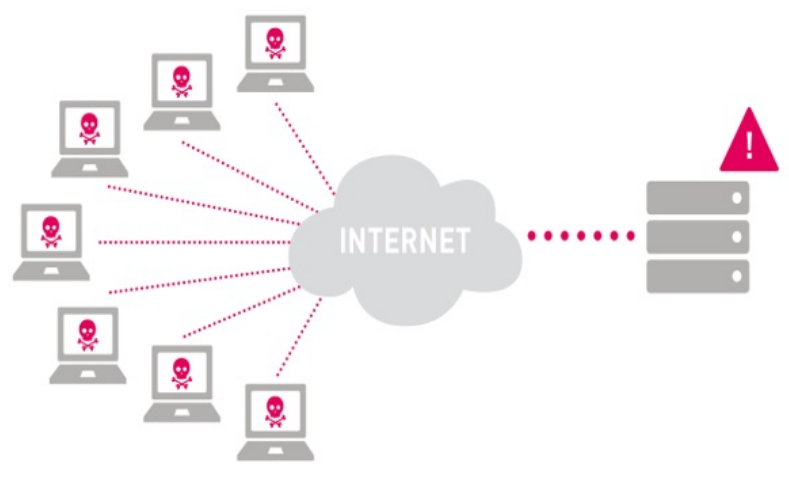

Figure 1: A topological network structure on which Distributed DoS attacks happen.

In Figure 1, it is shown that there are seven cyber attackers located on the left-hand side, sending a large number of unnecessary packets to three servers located on the right-hand side at the same time. Here, we assume that all cyber attackers give offence to the three servers through the Internet. Then, the network components which the cyber attackers target can be classified into the following:

- Type 1. Server targeting DoS attacks:

In Figure 2, it is shown that the seven cyber attackers give offence to one of three servers. Though only one server, which is encircled in blue, suffers from network traffic congestion happening on the interface of this server, the other two servers can continue to offer their service through the Internet.

- Type 2. Bottlenecked link targetting attacks.

In Figure 3, it is shown that the seven cyber attackers give offence to the bottlenecked link, which is encircled in green, connecting 


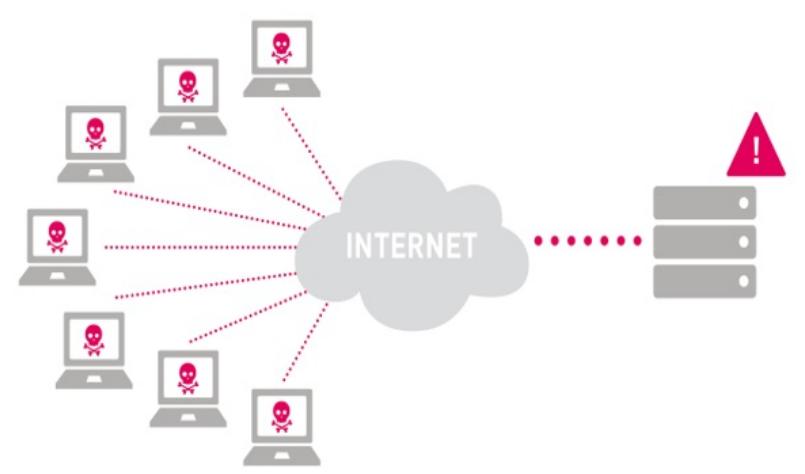

Figure 2: Cyber attackers targeting one of three servers located on the mid-part of the right-hand side.

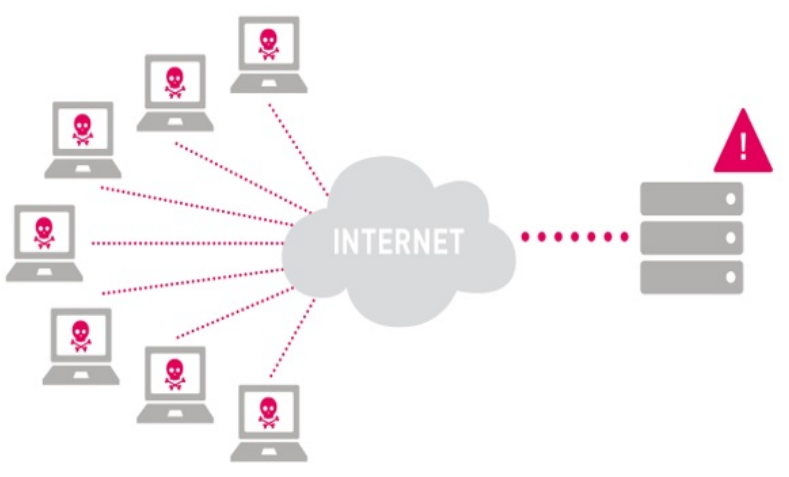

Figure 3: Cyber attackers targeting the bottlenecked link connecting between the Internet and the group which is composed of three servers.

between the group of all three servers and the Internet. Therefore, the network segment in which all three servers exist has been isolated from the Internet, and eventually, none of these servers can continue to offer their service to the clients through the Internet.

\section{ROUTING LOOPS AND PACKET OSCILLATIONS}

In this section, we explain that the topological difference between the routing loops and packet oscillations. First, an example of the routing loop can be illustrated as the following:

In Figure 4. The flow of packets streaming around clockwise on the loop composed of three routers are illustrated with the red colored arrow. The modern dynamic routing protocols are constructed so as to prevent such a packet streaming shown in this figure. Second, an example of packet oscillations can be illustrated as the following: In Figure 5. The flow of packets commuting between the both ends of the link located on the left-hand side are illustrated with two black colored arrows.

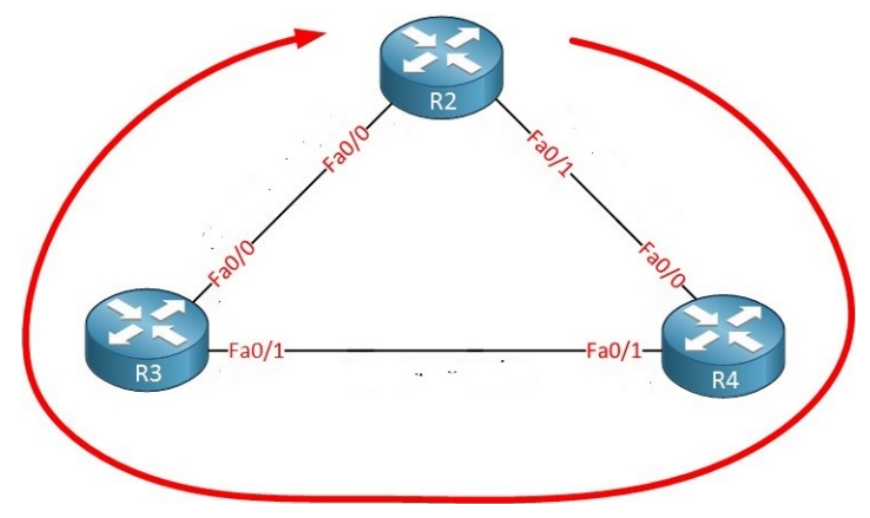

Figure 4: An example of the routing loop

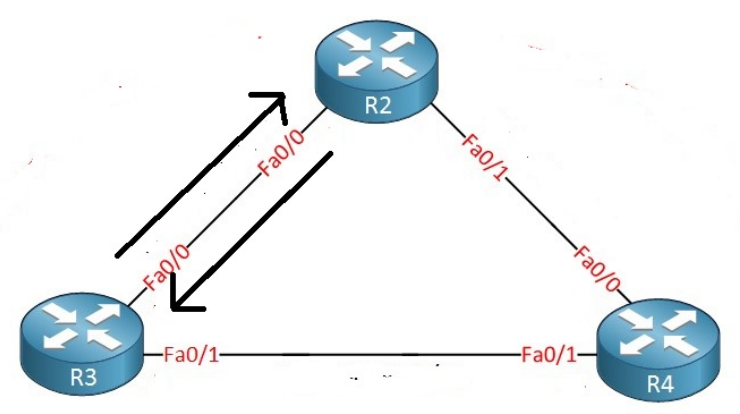

Figure 5: An example of packets oscillations.

\section{BOTTLENECKED LINK TARGETING ATTACS FROM REMOTE NETWORK SEGMENTS}

In this section, we show that the network traffic congestions brought about with routing loops and network traffic congestions brought about with the packet oscillations are different from each other, if we apply simultaneous combined use of dynamic routing with static one to network design. For example, the links connecting default gateways and the entrances of internet service providers must be configured with static routing, and all the network segments located behind the default gateways must be configured with dynamic routing. This configuration requires network administrators to use the dynamic routing combined with static routing simultaneously.

Though it is hardly possible to construct network segments on which packets commute on condition that the networks are constructed with dynamic routing protocols only, the simultaneous combined use of dynamic routing with static one brings about the packet oscillations as stated above. The simplest network structure on which intentional packet oscillations may happen is illustrated as the following: 


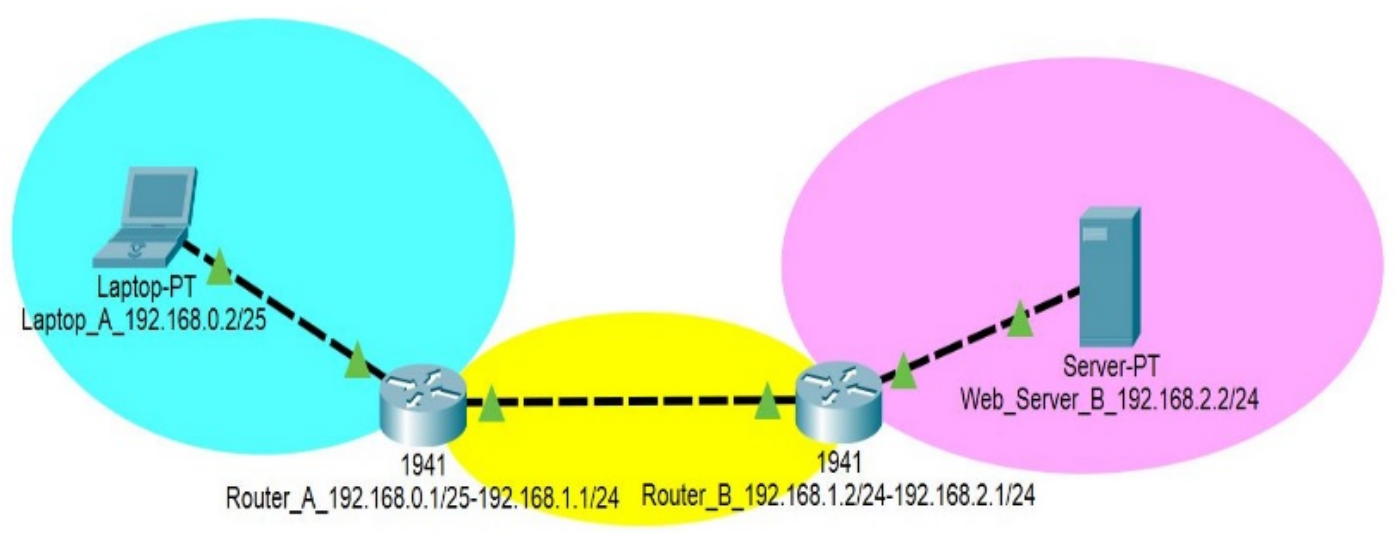

Figure 6: The simplest network structure on which packet oscillations can happen intentionally.

In Figure 6, it is illustrated that Laptop_A with its IP address 192.168.0.2/24, which is located on the left-hand side and encircled in blue, visits Web_Server_B with its IP address 192.168.2.2/24, which is located on the right-hand side and encircled in red, through the network segment with its network address 192.168.1.0/24, which is located in the central part and encircled in yellow, Exactly speaking, the network structure composed of the four network instruments are configured as the following:

- Router_A is designated as the default gateway for Laptop_A and is configured with the default-route forwarding any other packets than what belongs to 192.168.2.0/24.

- Router_B is designated as the default gateway for Web_Server_B and is configured with the static route forwarding all packets which is bound for the destinations belongs to $192.168 .0 .0 / 24$.

Under the assumptions as stated above, The routing path for the ICMP packets originating in Web_Server_B which are bound for Laptop_A can be recorded as the following:

Here, when we send some other ICMP packets which are bound for a non-existing PC for which 192.168.0.129 is assumed to be assigned, we can obtain the following:

In Figure 8, we can see the packet oscillation in the form of endless commutation between the gateway router of Laptop_A and that of Web_Server_B. The reason why such a phenomenon has happened can be clarified by the following:

- Step 1. Web_Server_B sends an ICMP packet whose final destination IP address is 192.168.0.129 to Router_B.

- Step 2. Router_B forwards the ICMP packets to Router_A, because the routing table of Router_B confirm that 192.168.0.129 belongs to 192.168.0.0/24.

- Step 3. After the ICMP packets has reached Router_A, Router_A tries to compare 192.168.0.129 with its network addresses which have been enrolled in Router_A and find that 192.168.0.129 belongs to neither 192.168.0.0/25 nor 192.168.1.0/24.
- Step 4. According to the default route configured in Router_A, the ICMP packet whose destination IP address is 192.168.0.129 forwards to Router_B. Therefore, the ICMP packet results in being forwarded back to Router_B, and ventually, this ICMP packet commutes between Router_A and Router_B, until the Time To Live assigned for the packet expires. .

If Web_Server_B sends another ICMP packet whose final destination IP address is 192.168.0.126 to Router_B, then another phenomenon which is different from what has happened as stated above. Since there does not exist any PC for which 192.168.0.126 is assigned, the ICMP packet cannot reach anywhere. But the routing path for the ICMP packet whose destination IP address is 192.168.0.129 is different from that for the ICMP packet whose destination IP address is $192.168,0.127$, which can be seen as the following:

The comparison of Figure 8 with Figure 9 clarifies that the difference between the routing path followed by a packet whose destination IP address is neither assigned for any PC nor contained in any network address and the routing path followed by a packet whose destination IP address is not assigned for any PC but included in a certain network address. More exactly speaking, while Figure 8 proves that there does not exist the network segment for which 192.168.0.128/25 is assigned, Figure 9 proves that there exists the network segment for which 192.168.0.0/25 is assigned, and the response in Figure 9 informing of the success in sending the ICMP packet whose destination IP address is 192.168 .1 .127 proves this IP address is the broadcast one of 192.168.0.0/25.

These results tell us that the difference as stated above enables cyber attackers located on remote network segments to investigate the correspondence between the network segments and the network addresses for which these segments are assigned from outside beyond the gateway routers. As for the protection against Distributed DoS attacks, we can refer to [5]. 


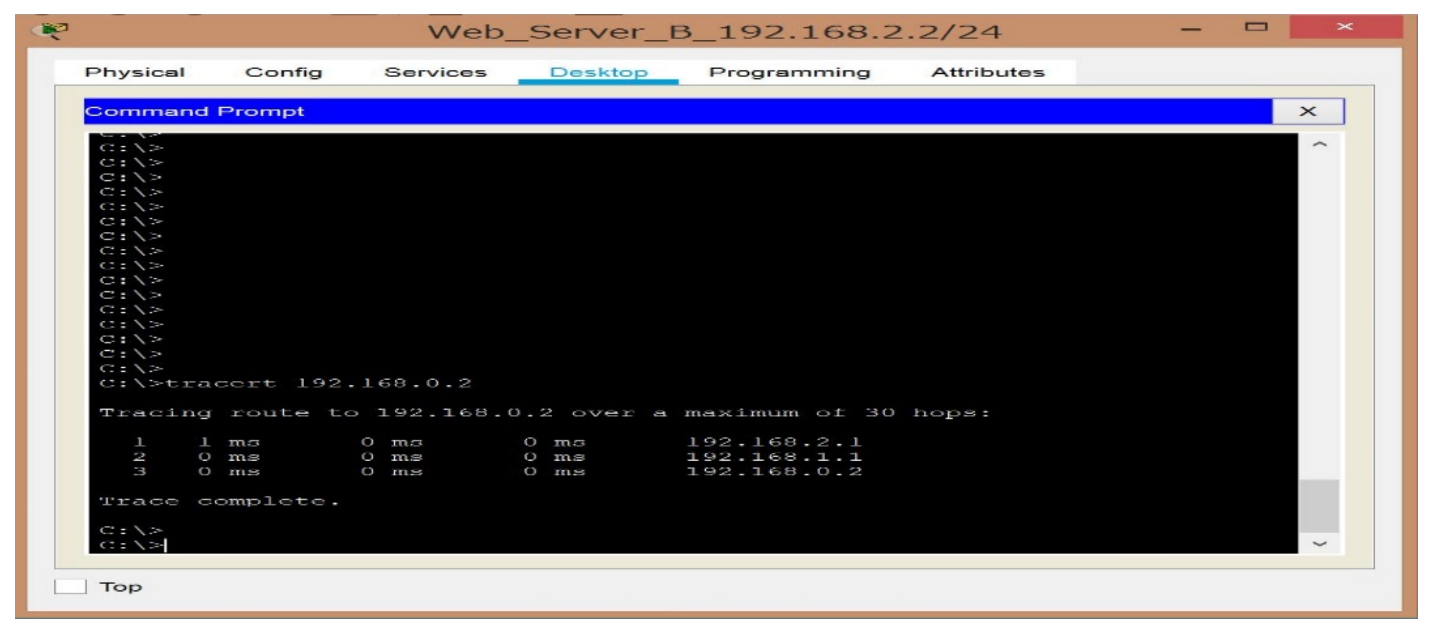

Figure 7: The routing path form Web_Server_B to Laptop_A through the transit routers.

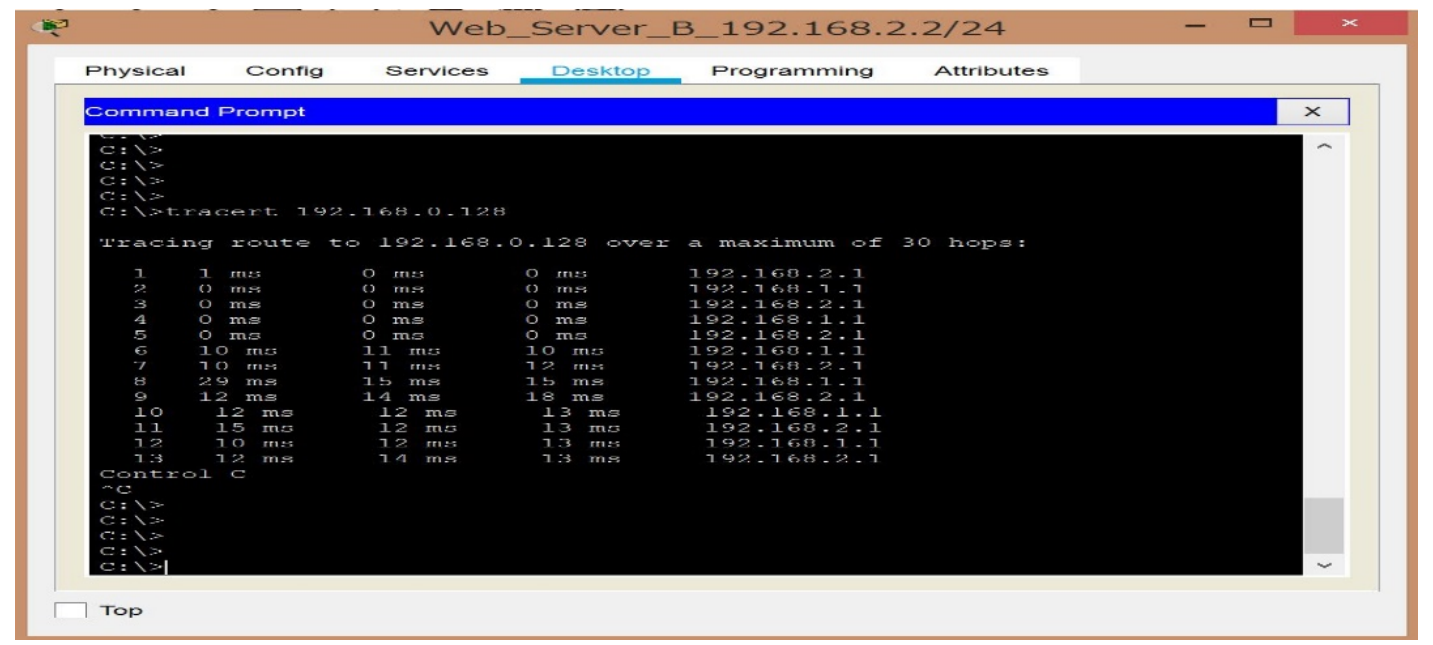

Figure 8: The packet oscillation between the gateway router for Web_Server_B and that for Laptop_A .

\section{SIMULTANEOUS COMBINED USE OF BOTTLENECKED LINK TARGETING ATTACKS WITH OTHER ATTACKS}

In the previous section, it is shown that network congestions can be brought about with the bottlenecked link targeting attacks from inside. In case that the cyber attackers share the network segment with the victims, they come to suffer from the same intentional network traffic congestions as the victims, because they must share not only the network segment but also the bottlenecked link with the victims. In this section, we discuss the problem asking if the bottlenecked link targeting attacks can be brought about by the cyber attackers existing in some other network segment which are disjoint from the network segment where the victims exist. For this purpose, it is considered that the cyber attackers must pay attention to sending the ICMP packets satisfying the following:

- The IP address assigned for the origin of the ICMP packets should pretend to be some other IP address.
- The IP address assigned for the destination of the ICMP packets should be none of already occupied address but belong to the network segment to which one end of the bottlenecked link is directly connected.

These conditions are necessary for the cyber attackers giving offence to the bottlenecked link from remote network segments and this is the reason why the network administrators should take precaution against DNS reflection attacks. Exactly speaking, the server reflection attacks, which is a malicious generalization of DNS Reflection Attacks, can be combined simultaneously with the bottlenecked link targeting attacks, and an example of the network structure can be illustrated as the following:

In Figure 10. There are two authenticated network segments, one of which is located on the left-hand side encircled in dark blue and the other of which is located on the right-hand side encircled in dark pink, respectively. Moreover, 192.168.0.0/24 and 192.168.1.0/24 are 


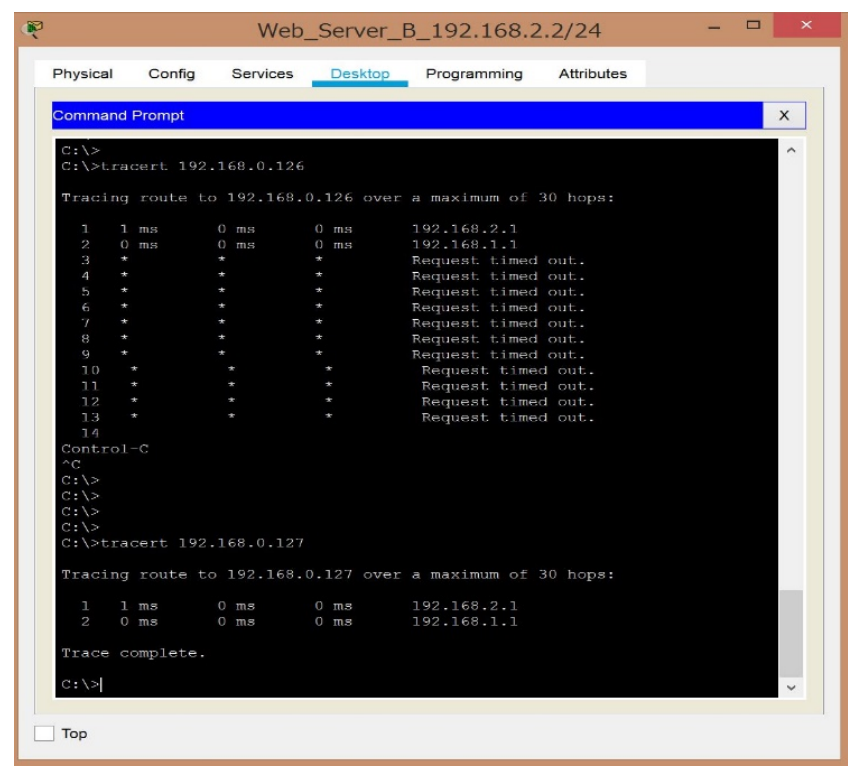

Figure 9: The routing path when an ICMP packet originating in Web_Server_B, which is bound for 192.168.0.127.

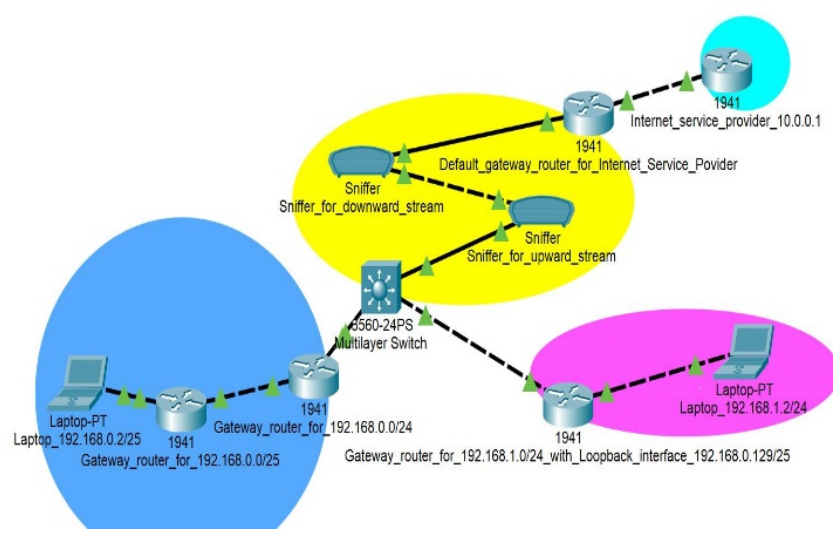

Figure 10: The network structure where the simultaneous combined use of bottlenecked link attacks with server reflection attacks.

assigned for the network segment encircled in dark blue and the network segment encircled in dark pink, respectively. Actually, as for 192.168.0.0/24, only 192.168.0.0/25, which occupies the former half of 192.168.0.0/24, is used, It is quite reasonable that the static route of 192.168.0.0/24 and that of 192.168.1.0/24 are configured in the routing table of Default_Gateway_for_Internet_Service_Provider. Besides the network structure as stated above, there are two sniffers located on the central area encircled in yellow, the upper one of which can capture the ICMP packets streaming downward and the lower one of which can capture the ICMP packets streaming upward. Here, if User_192.168.1.2/24 who is located on the network segment encircled in dark pink can operate not only his PC but Gateway_router_for
192.168.1.0/24_with_Loopback_Interface_192.168.1.129/25, then simultaneous combined use of the extended ping with the setting of virtual interfaces enables his PC to pretend to be Internet Service Provider by the following way:

- Step 1. User_192.168.1.2/24 activates a new virtual interface on the gateway router, for which 192.168.0.129/25 is assigned. This can be easily realized by making loopback interfaces.

- Step 2. User_192.168.1.2/24 send the ICMP packet which originates in 192.168.0.129/25 and is bound for Internet_Service_Provider for which 10.0.0.1/24 is assigned.

- Step 3. After the ICMP packet has reached Internet_Service _Provider, this router replies with another ICMP packets which originates in 10.0.0.1/24 and is bound for 192.168.0.129

- Step 4. The ICMP packet having originated in Internet _Service_Provider commutes between Default_Gateway_ Router_for_Internet_Service_Provider and Multilayer_ Switch.

Though only one ICMP packet has been sent to Internet Service_Provider, the packet oscillation is brought about by the reply from Internet_Service_Provider. The following two figures prove that the reply originating in 10.0.0.1 and being bound for 192.168.0.129 commutes between Default_Gateway_for_Internet_Service_Provider and Multilayer_Switch.

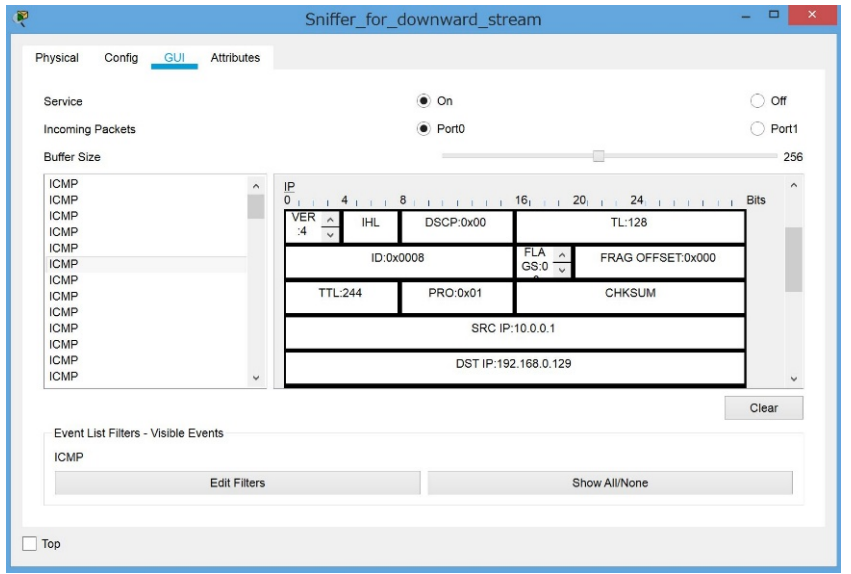

Figure 11: The packet oscillation captured by Sniffer_for_downward_stream,

In Figure 11, it is shown that the ICMP packet originating in 10.0.0.1 and being bound for 192.168.0.129 moves down.

In Figure 12, it is shown that the ICMP packet originating in 10.0.0.1 and being bound for 192.168.0.129 moves up. These two figures show, when Default_Gateway_Router_for

Internet_Service_Provider receives the ICMP packet originating in 10.0.0.1 and being bound for 192.168.0.129, this packet is forwarded to Multilayer_Switch according to the static route indicating that all the packets belonging to 192.168.0.0/24 should be forwarded to Multilayer_Switch. Actually, after this ICMP packet has reached Multilayer_Switch, it turns back to the link along which it has come, because the dynamic routing table of Multilayer_Switch is composed of 192.168.0.0/25 and 192.168.1.0/24, 


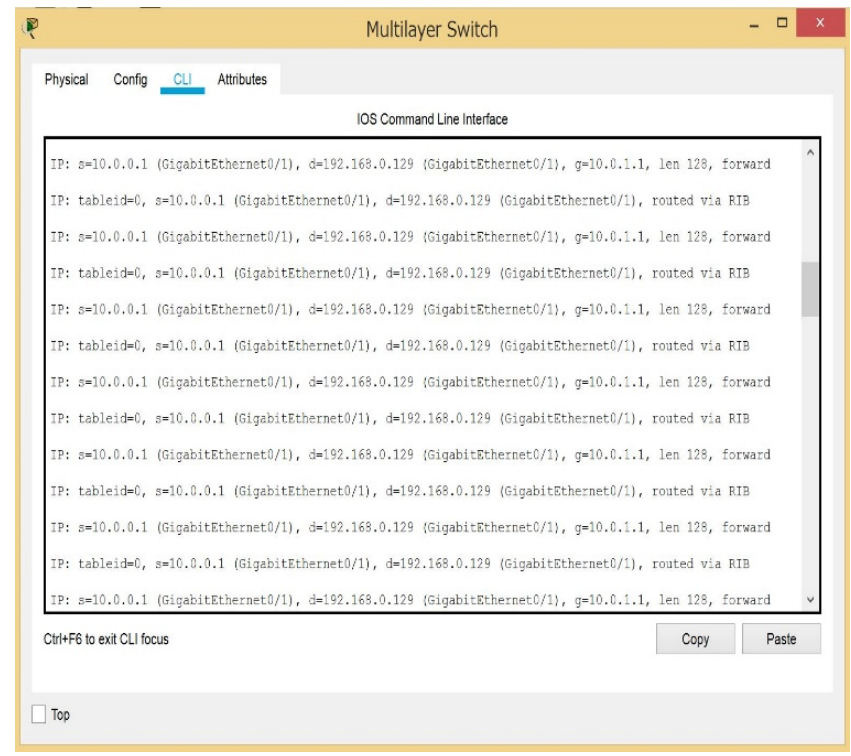

Figure 13: The packet oscillation captured by Multilayer_switch.,

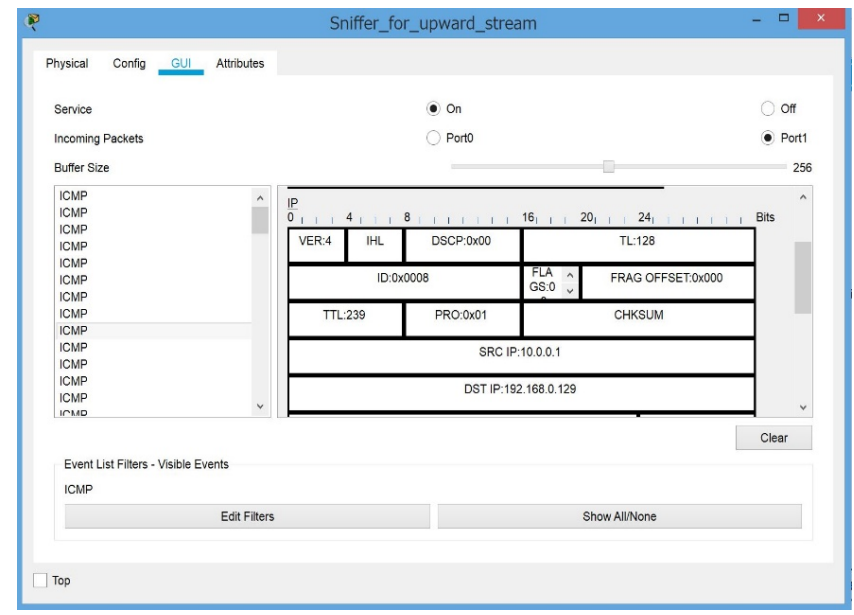

Figure 12: The packet oscillation captured by Sniffer_for_upward_stream,

the former address of which is advertised by Gateway_router_for -192.168.0.0/24 and the latter address of which is advertised by Gatway_router_for_192.168.1.0/24. Since 192.168.0.128/25 has not been used yet, Gateway_router_for_192.168.0.0/24 is not advertised to Multilayer_switch, the ICMP packet originating in 10.0.0.1 and being bound for 192.168.0.129 is sent back to Gateway_router_for_Internet_Service_Provider along the bottlenecked link connecting between Multilayer_switch and Default_Gateway_Router_for_Internet_Service_Provider.

Even if the network administrators do not use both sniffers for capturing sequential movement of packets, the debugging being activated on Multilayer_switch can capture the movement as the following:

In Figure 13, the output obtained by application of Debugging to Multilayer_Switch is recorded. There are so many ICMP packets originating in 10.0.0.1 and being bound for 192.168.0.129 in the monitor of Multilayer_Switch. Unfortunately, we cannot answer the question asking whether this congestion results from the server targeting attacks or the bottlenecked link targeting ones with this output only.

As for the maliciously revised version of DoS attacks, we can refer to [6] and [7].

\section{CONCLUSIONS}

In this paper, it is shown that Distributed DoS attacks discriminate themselves from other cyber attacks in that it is difficult to discriminate the spontaneous network traffic congestions from the intentional network traffic congestions, and that the simultaneous combined us of the bottlenecked link targeting attacks with server reflection attacks enables the cyber attackers to pretend to be other authenticated network users.

As this consideration tells us that, the more cyber attacks diversify, the more complicated is the joint combination of cyber attacks. Therefore, the classification of cyber attacks will play so important roles in preventing the hybrid attacks from being produced and in specifying the network skills used maliciously for cyber attacks.

\section{REFERENCES}

[1] O. Santos and J. Muniz, CCNA Cyber Ops Secfnd 210-250, Cisco Press, Indianapolis, 1st edition, 2017.

[2] O. Santos and J. Muniz, CCNA Cyber Ops Secops 210-255, Cisco Press, Indianapolis, 1 st edition, 2017.

[3] D. E. Knuth, The Art of Computer Programming, Addison-Wesley Publishing Company, Massachusetts, 2nd edition, 1973.

[4] S. Akashi and Y. Tong, "Classification of DHCP Spoofing and Effectiveness of DHCP Snooping," Proceedings on 2018 International Conference on Advances in Computer Technology, Information science and Communication, edited by Wen-Bing Horng and Yong Yue, pp. 233-238, ISBN:978-989-758-357-5, 2019.

[5] G. Loukas, G. Oke, "Protection Against Denial of Service Attacks: A Survey", Comput. J. vol.53(2010), no.7, pp. 1020-1037, doi:10.1093/comjnl/bxp078.

[6] Y. Chen; K. Hwang, Y-K Kwok, "Filtering of shrew DDoS attacks in frequency domain", The IEEE Conference on Local Computer Networks 30th Anniversary (LCN'05) vol.l, pp. 8, doi:10.1109/LCN.2005.70. ISBN 978-0-7695-2421-4.

[7] U. Ben-Porat, A. Bremler-Barr, H. Levy, "Vulnerability of Network Mechanisms to Sophisticated DDoS Attacks". IEEE Transactions on Computers,vol.62(2013),no.5,pp.1031-1043, doi:10.1109/TC.2012.49, ISSN 0018-9340 